\title{
Realizing Tunable White Light Emission in Lead-Free Indium (III) Bromine Hybrid Single Crystals through Antimony (III) Cation Doping
}

Zhongyuan $\mathrm{Li}_{1}^{\dagger}{ }^{\dagger}$ Gaomin Song, ${ }^{,, \S, l}$ Ye Li, ${ }^{*}, \dagger$ Le Wang, ${ }^{*, \perp}$ Tianliang Zhou, ${ }^{\dagger}$ Zheshuai Lin, ${ }^{\ddagger, \S, l}$ and Rong-Jun Xie ${ }^{*} \dagger$

${ }^{\dagger}$ College of Materials, State Key Laboratory of Physical Chemistry of Solid Surfaces and Fujian Province Key Laboratory of Materials Genome, Xiamen University, Simingnan Road 422, Xiamen 361005, P. R. China

${ }_{\ddagger}^{\ddagger}$ Technical Institute of Physics and Chemistry, Chinese Academy of Sciences, Beijing 100190, P. R. China

§University of Chinese Academy of Sciences, Beijing 100190, P. R. China

"Center of Materials Science and Optoelectronics Engineering, University of the Chinese Academy of Sciences, Beijing 100049, P. R. China

${ }^{\perp}$ College of Optical and Electronic Technology, China Jiliang University, Hangzhou 310018, Zhejiang, P.R. China 


\section{EXPERIMENTAL SECTION}

\section{Materials.}

Indium (III) bromide $\left(\mathrm{InBr}_{3}, 99.99 \%\right)$ and Antimony bromide $\left(\mathrm{SbBr}_{3}, 99.99 \%\right)$ were purchased from Alfa Aesar. $\mathrm{C}_{6} \mathrm{H}_{5} \mathrm{CH}_{2} \mathrm{CH}_{2} \mathrm{NH}_{3} \mathrm{Br}$ (98\%) was purchased from Xi'an Polymer Light Technology Corp. Hydrobromic acid $\left(\mathrm{HBr}, 48\right.$ wt.\% in $\left.\mathrm{H}_{2} \mathrm{O}\right)$ was purchased from Aladdin. All chemicals were used as received unless otherwise.

\section{Synthesis.}

Bulk single crystals of $\left(\mathrm{C}_{8} \mathrm{NH}_{12}\right)_{6} \mathrm{InBr}_{9} \cdot \mathrm{H}_{2} \mathrm{O}$ were grown by slowly cooling a saturated hot $\mathrm{HBr}$ solution with stoichiometric amounts of $\mathrm{InBr}_{3}$ and $\mathrm{C}_{8} \mathrm{NH}_{12} \mathrm{Br}$. Specifically, 0.25 $\mathrm{mmol} \mathrm{InBr}_{3}$ and $1.5 \mathrm{mmol} \mathrm{C}_{8} \mathrm{NH}_{12} \mathrm{Br}(1: 6$ molar ratio $)$ were respectively added into $3 \mathrm{ml} \mathrm{HBr}$ solution. The mixture was heated and stirred at $100^{\circ} \mathrm{C}$ for $0.5 \mathrm{~h}$ until the solution turned clear. Then the solution was transferred to a $100{ }^{\circ} \mathrm{C}$ oven immediately and cooled to $45^{\circ} \mathrm{C}$ at $2.5^{\circ} \mathrm{C} / \mathrm{h}$ without stirring. Colorless rectangular rod-like crystals precipitated during slow cooling (Figure S1). Bulk crystals were washed with the $\mathrm{HBr}$ solution three times, then they were collected and dried in a vacuum oven at $65^{\circ} \mathrm{C}$ overnight. The yield was calculated at $~$ $75 \%$ based on the In content. The synthetic procedure of Sb-doped $\left(\mathrm{C}_{8} \mathrm{NH}_{12}\right)_{6} \mathrm{InBr}_{9} \cdot \mathrm{H}_{2} \mathrm{O}$ single crystals was similar to that of pure $\left(\mathrm{C}_{8} \mathrm{NH}_{12}\right)_{6} \mathrm{InBr}_{9} \cdot \mathrm{H}_{2} \mathrm{O}$ with the addition of $\mathrm{SbBr}_{3}$ in the $\mathrm{HBr}$ solution. The nominal amount of $\mathrm{SbBr}_{3}(\mathrm{n} \mathrm{mmol})$ is based on $\left(\mathrm{C}_{8} \mathrm{NH}_{12}\right)_{6} \operatorname{In}_{1}$. ${ }_{x} \mathrm{Br}_{9} \cdot \mathrm{H}_{2} \mathrm{O}: x \mathrm{Sb}$, where the range of $x$ is from 0.01 to $25 \%$. The yield was calculated as above $75 \%$ based on totally the $\mathrm{In}$ and $\mathrm{Sb}$ contents. Powders of series $\left(\mathrm{C}_{8} \mathrm{NH}_{12}\right)_{6} \mathrm{InBr}_{9} \cdot \mathrm{H}_{2} \mathrm{O}: x \mathrm{Sb}$ for characterizations were obtained via grinding bulk single crystal thoroughly and then placing them into an oven at $100^{\circ} \mathrm{C}$ for 2 hours.

\section{Characterizations.}

Single crystal X-ray diffraction (SCXRD) measurements were conducted on an Oxford Gemini S Ultra system with graphite-monochromated Mo Ka radiation $(\lambda=0.71073 \AA)$ at $100 \mathrm{~K}$. The multi-scan absorption corrections were applied by using the program CrysAlis. The crystal structure was solved and refined by least squares on $\mathrm{F}^{2}$ using the SHELXTL and OLEX ${ }^{2}$ programs. The VESTA software was used to depict crystal structure. Simulated XRD pattern was calculated from SCXRD and extracted from VESTA software. The powder X-ray 
diffraction (PXRD) analysis was performed using a Bruker, D8 ADVANCE with $\mathrm{Cu} \mathrm{Ka}(\lambda=$ $1.5406 \AA$ ) operating at $40 \mathrm{kV} / 40 \mathrm{~mA}$. Optical images under ambient light were obtained by widefield microscopes (Leica LAS X). Fluorescence microscopy images under $365 \mathrm{~nm} \mathrm{UV}$ light were obtained with an Olympus DP74 color fluorescence microscope camera. SEM images together with EDS spectra were obtained via a field-emission scanning electron microscope (SEM, Hitachi, SU70). The elemental analysis of C, H and $\mathrm{N}$ was performed on the Vario EL III analyzer. Inductively coupled plasma optical emission spectrometer measurements (ICP-OES) were performed on Optima 5300DV (PerkinElmer Inc.). TGA measurements were conducted on a thermal analyzer (NETZSCH, TG 209 F 1$)$. The samples were heated from 35 to $840{ }^{\circ} \mathrm{C}$ with at a rate of $10{ }^{\circ} \mathrm{C} \mathrm{min}-1$ under a $\mathrm{N}_{2}$ flux of $40 \mathrm{~mL} \mathrm{~min}^{-1}$. Fourier transform infrared (FTIR) spectra were recorded from 500 to $4000 \mathrm{~cm}^{-1}$ using a Thermo Scientific Nicolet iS10 FT-IR spectrometer (Waltham, USA). Optical diffuse reflectance measurements were carried out using a Shimadzu UV-3600 UV-vis-NIR spectrometer operating in the $200-800 \mathrm{~nm}$ region at room temperature. Steady-state photoluminescence spectra were obtained on an Edinburgh Instruments FLS980 spectrophotometer at room temperature. Time-resolved spectra measurements were collected using $\mu \mathrm{F} 920 \mathrm{H}$ pulsed xenon flashlamp as the excitation source with a pulse repetition rate of $100 \mathrm{~Hz}$. Temperature-dependent emission and decay data were collected using the FLS980 spectrophotometer equipped with a closed-cycle cryostat (DE202, Advanced Research Systems) at a series of temperature from 10 to $300 \mathrm{~K}$. The powerdependent photoluminescence spectra were measured using the $375 \mathrm{~nm}$ (LE-LS-375140TFCA, 1-140 mW) laser. Absolute PLQE measurements were performed at room temperature by using an integrated sphere on Hamamatsu Quantaurus-QY system (C11347$11)$.

\section{Computational Methods.}

The first-principle calculations were performed using the plane-wave pseudopotential method implemented in the CASTEP package. The exchange-correlation functional was chosen as the Perdew-Burke-Emzerhof (PBE) functional within the generalized gradient approximation (GGA). ${ }^{1-2}$ The k-point separation was set as gamma point only in the Brillouin 
zone leading to corresponding Monkhorst-Pack k-point meshes of $1 \times 1 \times 1 .^{3}$ And optimized ultra-soft pseudopotentials are adopted in these calculations. The choice of these computational parameters is good enough to ensure the accuracy of present purpose. 


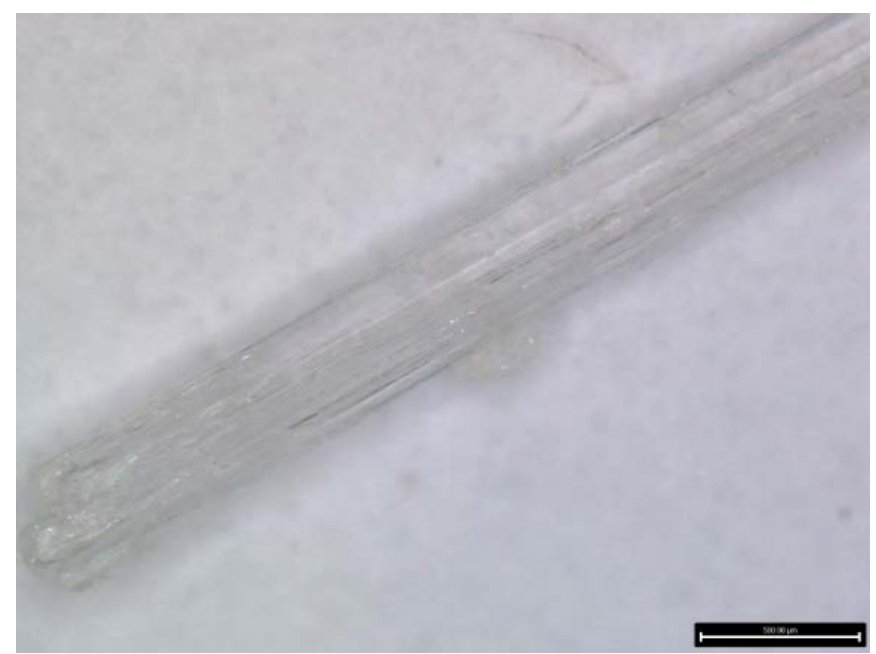

Figure S1. Optical image of $\left(\mathrm{C}_{8} \mathrm{NH}_{12}\right)_{6} \mathrm{InBr}_{9} \cdot \mathrm{H}_{2} \mathrm{O}$ single crystal under natural light (the scale bar is $500 \mu \mathrm{m}$ ).

Table S1. Single Crystal X-ray data for $\left(\mathrm{C}_{8} \mathrm{NH}_{12}\right)_{6} \mathrm{InBr}_{9} \cdot \mathrm{H}_{2} \mathrm{O}$.

\begin{tabular}{|c|c|}
\hline Identification code & $\left(\mathrm{C}_{8} \mathrm{NH}_{12}\right)_{6} \operatorname{InBr}_{9} \cdot \mathrm{H}_{2} \mathrm{O}$ \\
\hline Empirical formula & $\mathrm{C}_{48} \mathrm{H}_{74} \mathrm{Br}_{9} \mathrm{InN}_{6} \mathrm{O}$ \\
\hline Formula weight & 1585.14 \\
\hline Temperature/K & $99.9(3)$ \\
\hline Crystal system & monoclinic \\
\hline Space group & P21 \\
\hline $\mathbf{a} / \AA$ & $10.29960(10)$ \\
\hline $\mathbf{b} / \AA ̊ \AA$ & $26.3489(2)$ \\
\hline $\mathbf{c} / \AA$ & $22.2194(2)$ \\
\hline $\mathfrak{a} /{ }^{\circ}$ & 90 \\
\hline$\beta /{ }^{\circ}$ & $92.3370(10)$ \\
\hline$\gamma /{ }^{\circ}$ & 90 \\
\hline Volume/Å3 & $6024.96(9)$ \\
\hline $\mathbf{Z}$ & 4 \\
\hline$\rho_{\text {calcg }} / \mathrm{cm}^{3}$ & 1.748 \\
\hline$\mu / \mathbf{m m}^{-1}$ & 10.417 \\
\hline $\mathbf{F}(\mathbf{0 0 0})$ & 3104.0 \\
\hline Crystal size $/ \mathbf{m m}^{3}$ & $0.12 \times 0.11 \times 0.1$ \\
\hline Radiation & $\mathrm{CuKa}(\lambda=1.54184)$ \\
\hline
\end{tabular}




\section{Index ranges}

Reflections collected

Independent reflections

Data/restraints/parameters

Goodness-of-fit on $\mathrm{F}^{2}$

Final $R$ indexes $[\mathrm{I}>=\mathbf{2 \sigma}(\mathrm{I})]$

Final $R$ indexes [all data]

Largest diff. peak/hole / e $\AA^{-3}$
$-12 \leq \mathrm{h} \leq 13,-33 \leq \mathrm{k} \leq 30,-28 \leq 1 \leq 22$

46080

$20598\left[\mathrm{R}_{\text {int }}=0.0491, \mathrm{R}_{\text {sigma }}=0.0575\right]$

$20598 / 265 / 1250$

1.063

$\mathrm{R}_{1}=0.0362, \mathrm{wR}_{2}=0.0867$

$\mathrm{R}_{1}=0.0418, \mathrm{wR}_{2}=0.0961$

$1.08 /-0.92$

$0.020(7)$

$R_{1}=\sum\left(\| F_{o}\left|-F_{c}\right|||\right) /\left|F_{o}\right|, \mathrm{wR}_{2}=\left\{\sum\left[\mathrm{w}\left(\mathrm{F}_{\mathrm{o}}^{2}-\mathrm{F}_{\mathrm{c}}^{2}\right)^{2}\right] / \sum\left[\mathrm{w}\left(\mathrm{F}_{\mathrm{o}}^{2}\right)\right]^{2}\right\}^{1 / 2}$

where $\mathrm{w}=1 /\left|\sigma^{2} \mathrm{~F}_{0}^{2}+(\mathrm{AP})^{2}+\mathrm{BP}\right|$, with $\mathrm{P}=\mathrm{F}_{0}^{2}+2 \mathrm{~F}_{\mathrm{c}}^{2} / 3$ and weight coefficients $\mathrm{A}$ and $\mathrm{B}$.

Table S2. Selected bond angles for $\left(\mathrm{C}_{8} \mathrm{NH}_{12}\right)_{6} \mathrm{InBr}_{9} \cdot \mathrm{H}_{2} \mathrm{O}$.

\begin{tabular}{|c|c|c|c|}
\hline Atom & Atom & Atom & Angle $/^{\circ}$ \\
\hline $\mathrm{Br} 2$ & In 1 & $\mathrm{Br} 1$ & $89.58(4)$ \\
\hline $\mathrm{Br} 2$ & $\operatorname{In} 1$ & $\mathrm{Br} 3$ & $89.26(3)$ \\
\hline $\mathrm{Br} 2$ & In 1 & Br4 & $178.62(4)$ \\
\hline $\mathrm{Br} 2$ & $\operatorname{In} 1$ & $\mathrm{Br} 5$ & $90.55(3)$ \\
\hline $\mathrm{Br} 2$ & $\operatorname{In} 1$ & Br6 & $91.21(3)$ \\
\hline $\mathrm{Br} 3$ & $\operatorname{In} 1$ & Br1 & $86.65(3)$ \\
\hline $\mathrm{Br} 4$ & In 1 & Br1 & $89.09(3)$ \\
\hline $\mathrm{Br} 4$ & $\operatorname{In} 1$ & Br3 & $90.30(3)$ \\
\hline $\mathrm{Br} 4$ & $\operatorname{In} 1$ & Br6 & $90.09(3)$ \\
\hline $\mathrm{Br} 5$ & $\operatorname{In} 1$ & $\mathrm{Br} 1$ & $90.99(3)$ \\
\hline $\mathrm{Br} 5$ & In 1 & Br3 & $177.63(4)$ \\
\hline $\mathrm{Br} 5$ & $\operatorname{In} 1$ & $\mathrm{Br} 4$ & $89.84(3)$ \\
\hline Br5 & In 1 & Br6 & $93.02(3)$ \\
\hline Br6 & $\operatorname{In} 1$ & Br1 & $175.91(4)$ \\
\hline Br6 & In 1 & Br3 & $89.35(3)$ \\
\hline Br7 & $\operatorname{In} 2$ & $\mathrm{Br} 8$ & $90.50(3)$ \\
\hline $\mathrm{Br} 7$ & $\operatorname{In} 2$ & $\mathrm{Br} 9$ & $91.74(3)$ \\
\hline Br7 & $\operatorname{In} 2$ & Br10 & $89.95(3)$ \\
\hline
\end{tabular}




\begin{tabular}{|c|c|c|c|}
\hline Br7 & $\operatorname{In} 2$ & Br11 & $89.21(3)$ \\
\hline $\mathrm{Br} 7$ & $\operatorname{In} 2$ & Br12 & $179.69(4)$ \\
\hline Br8 & $\operatorname{In} 2$ & Br10 & $179.51(4)$ \\
\hline Br9 & $\operatorname{In} 2$ & $\mathrm{Br} 8$ & $90.15(3)$ \\
\hline Br9 & $\operatorname{In} 2$ & Br10 & $89.65(3)$ \\
\hline Br11 & $\operatorname{In} 2$ & $\mathrm{Br} 8$ & $89.27(3)$ \\
\hline Br11 & $\operatorname{In} 2$ & $\mathrm{Br} 9$ & $178.89(4)$ \\
\hline Br11 & $\operatorname{In} 2$ & Br10 & $90.92(3)$ \\
\hline Br12 & $\operatorname{In} 2$ & $\mathrm{Br} 8$ & $89.81(3)$ \\
\hline Br12 & $\operatorname{In} 2$ & $\mathrm{Br} 9$ & $88.22(3)$ \\
\hline $\operatorname{Br} 12$ & $\operatorname{In} 2$ & Br10 & $89.74(3)$ \\
\hline $\mathrm{Br} 12$ & $\operatorname{In} 2$ & Br11 & $90.83(3)$ \\
\hline
\end{tabular}

Table S3. Selected bond lengths for $\left(\mathrm{C}_{8} \mathrm{NH}_{12}\right)_{6} \mathrm{InBr}_{9} \cdot \mathrm{H}_{2} \mathrm{O}$.

\begin{tabular}{|c|c|c|}
\hline Atom & Atom & Length/Å \\
\hline In 1 & $\mathrm{Br} 1$ & $2.7270(11)$ \\
\hline In 1 & $\mathrm{Br} 2$ & $2.6346(11)$ \\
\hline In 1 & $\mathrm{Br} 3$ & $2.7055(10)$ \\
\hline In 1 & $\mathrm{Br} 4$ & $2.6713(11)$ \\
\hline $\operatorname{In} 1$ & $\mathrm{Br} 5$ & $2.6475(10)$ \\
\hline In 1 & Br6 & $2.6878(11)$ \\
\hline In 2 & $\mathrm{Br} 7$ & $2.6419(10)$ \\
\hline In 2 & Br8 & $2.6951(11)$ \\
\hline In 2 & $\mathrm{Br} 9$ & $2.6731(10)$ \\
\hline In 2 & Br10 & $2.7053(11)$ \\
\hline In 2 & Br11 & $2.6704(11)$ \\
\hline In 2 & $\mathrm{Br} 12$ & $2.6660(10)$ \\
\hline
\end{tabular}




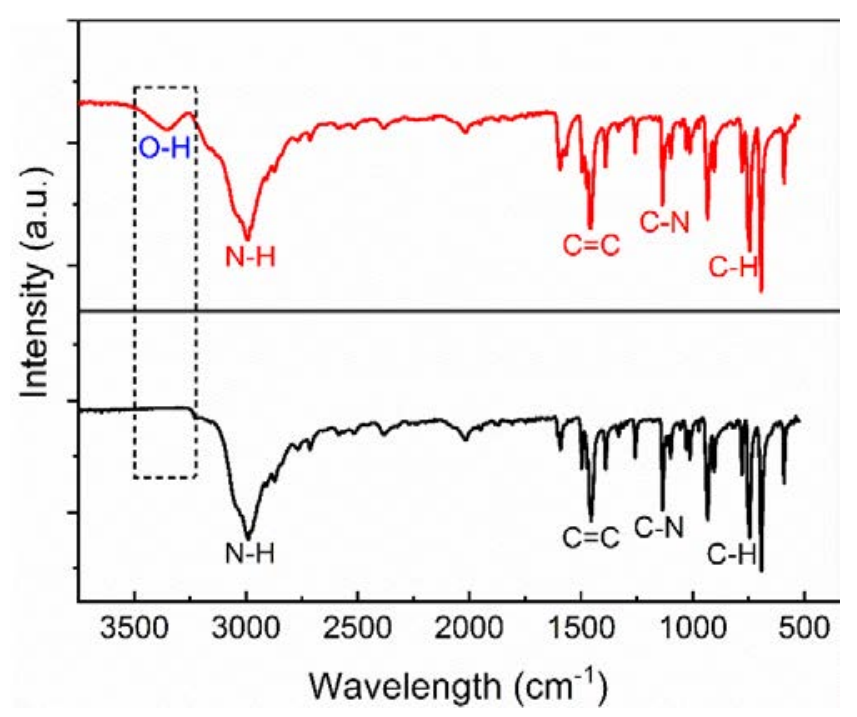

Figure S2. FT-IR spectra of $\left(\mathrm{C}_{8} \mathrm{NH}_{12}\right)_{6} \mathrm{InBr}_{9} \cdot \mathrm{H}_{2} \mathrm{O}$ and $\mathrm{C}_{8} \mathrm{NH}_{12} \mathrm{Br}$.

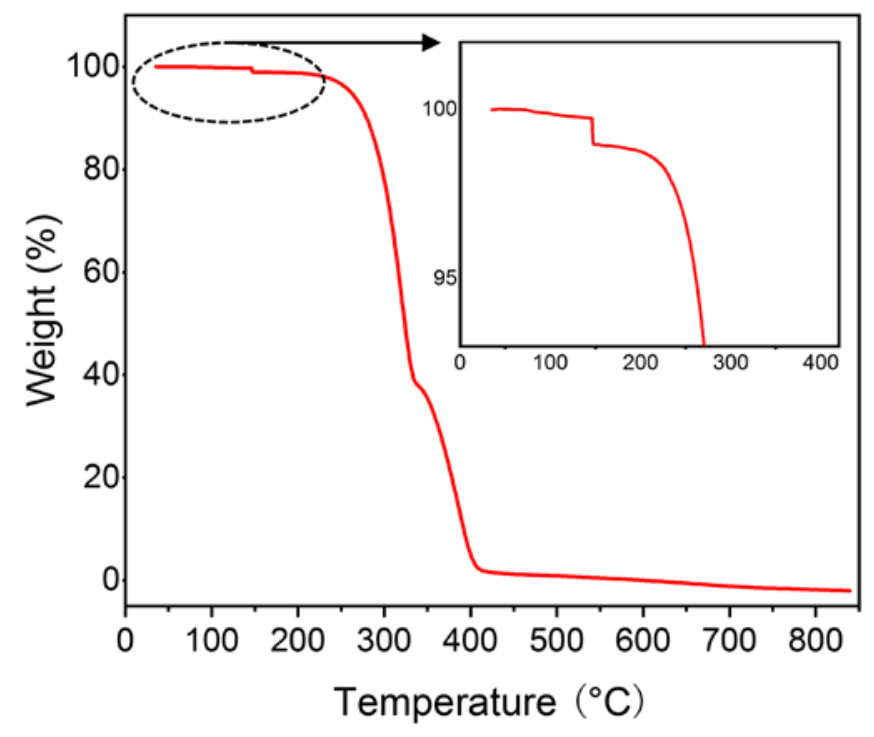

Figure S3. TG curves of $\left(\mathrm{C}_{8} \mathrm{NH}_{12}\right)_{6} \mathrm{InBr}_{9} \cdot \mathrm{H}_{2} \mathrm{O}$.

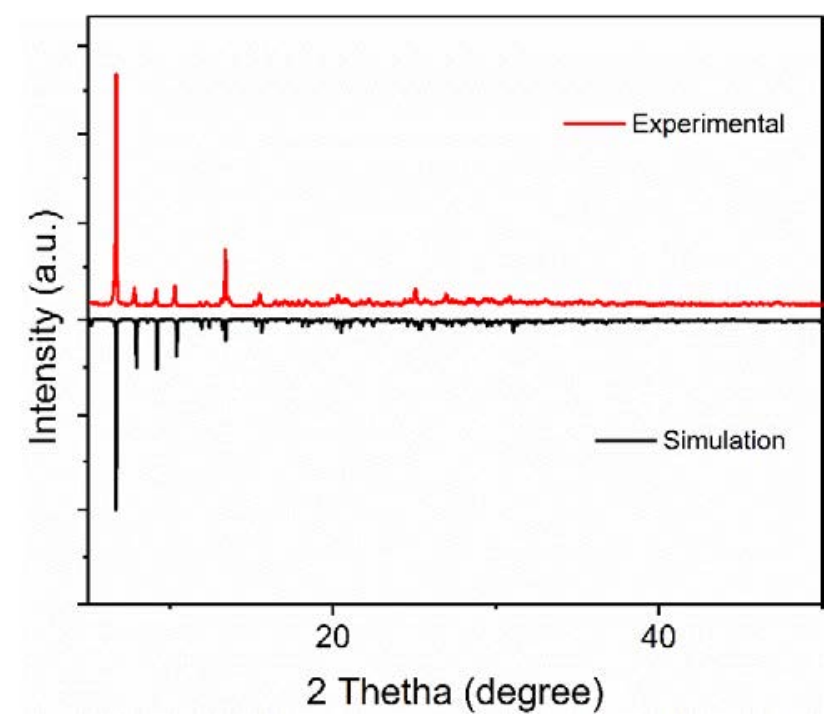

Figure S4. PXRD and SCXRD patterns of $\left(\mathrm{C}_{8} \mathrm{NH}_{12}\right)_{6} \mathrm{InBr}_{9} \cdot \mathrm{H}_{2} \mathrm{O}$. 
Table S4. Element analysis of C, H and N via Vario EL III analyzer and SCXRD.

\begin{tabular}{ccc}
\hline Element & Calculation (\%) & Experiment (\%) \\
\hline $\mathrm{C}$ & 36.34 & 35.87 \\
$\mathrm{H}$ & 4.54 & 4.38 \\
$\mathrm{~N}$ & 5.29 & 5.26 \\
\hline
\end{tabular}

Table S5. Element analysis of In and Br via EDS and SXCRD.

\begin{tabular}{ccc}
\hline Element & Calculation (atom\%) & Experiment (atom\%) \\
\hline In & 90 & 90.07 \\
$\mathrm{Br}$ & 10 & 9.93 \\
\hline
\end{tabular}

Table S6. The data of $\mathrm{Sb}$ mole percent $(\mathrm{mol} \%)$ in (PEA) ${ }_{6} \mathrm{InBr}_{9} \cdot \mathrm{H}_{2} \mathrm{O}: x \mathrm{Sb}(x=0.05,5,25 \%)$ measured via ICPOES.

\begin{tabular}{cccc}
\hline $\begin{array}{c}\text { Nominal Sb molar percent } \\
(\mathbf{m o l} \%)\end{array}$ & $\begin{array}{c}\text { In content } \\
(\mathbf{m g} / \mathbf{L})\end{array}$ & $\begin{array}{c}\text { Sb content } \\
(\mathbf{m g} / \mathbf{L})\end{array}$ & $\begin{array}{c}\text { Actual Sb molar percent } \\
(\mathbf{m o l} \%)\end{array}$ \\
\hline 0.05 & 0.0131 & 45.9400 & 0.0269 \\
5 & 1.5140 & 37.8430 & 4.3625 \\
25 & 2.3714 & 0.6412 & 20.2321 \\
\hline
\end{tabular}

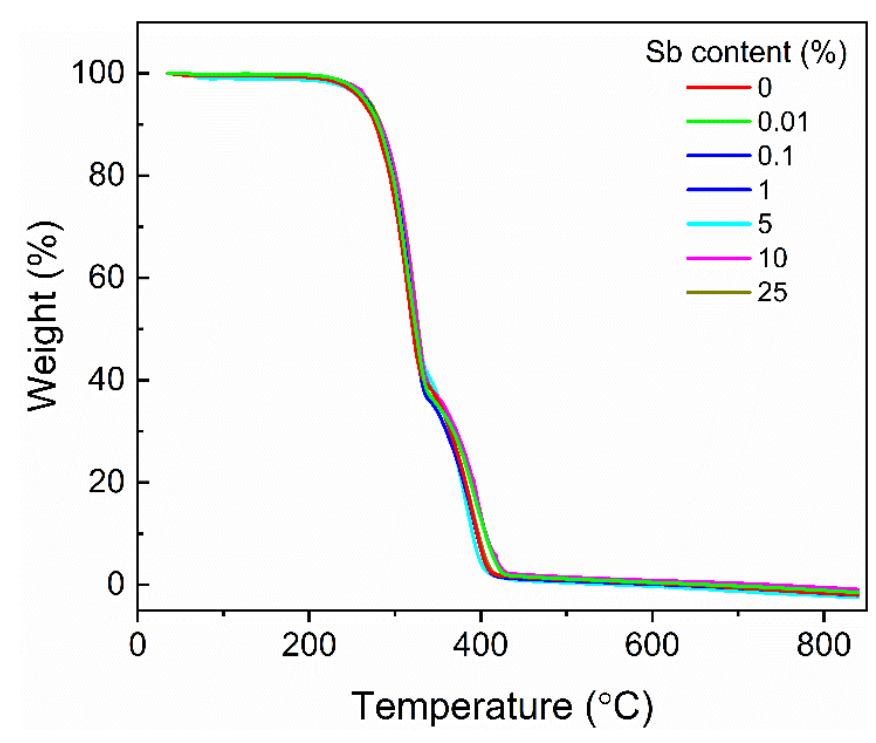

Figure S5. TG curves of $\left(\mathrm{C}_{8} \mathrm{NH}_{12}\right)_{6} \mathrm{InBr}_{9} \cdot \mathrm{H}_{2} \mathrm{O}: x \mathrm{Sb}(x=0 \sim 25 \%)$. 


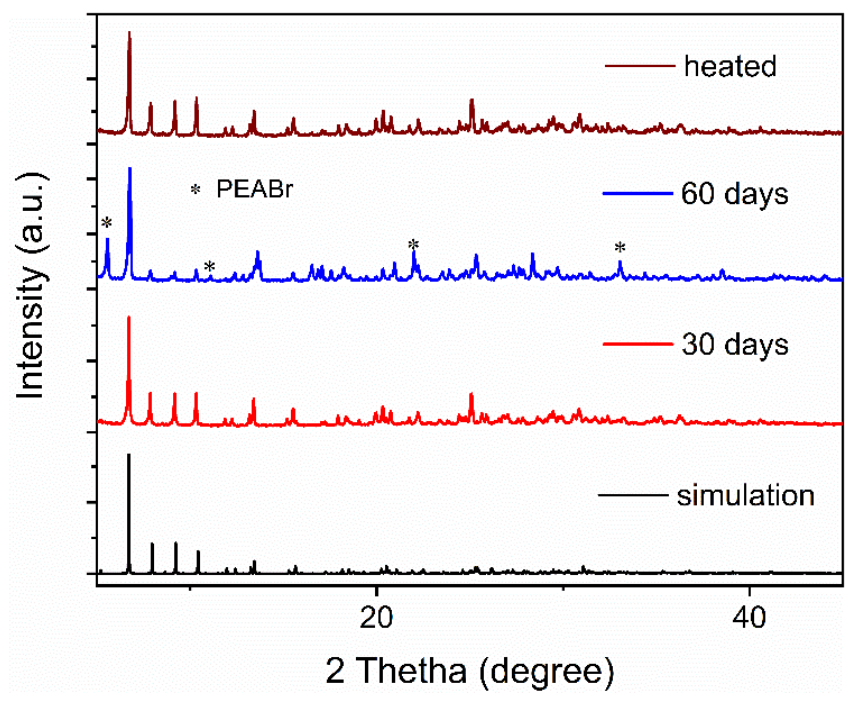

Figure S6. PXRD patterns of $\left(\mathrm{C}_{8} \mathrm{NH}_{12}\right)_{6} \mathrm{InBr}_{9} \cdot \mathrm{H}_{2} \mathrm{O}: 0.1 \% \mathrm{Sb}$ after exposure to 30 days and 60 days and followed by heating at $100^{\circ} \mathrm{C}$ in air for 2 hours.
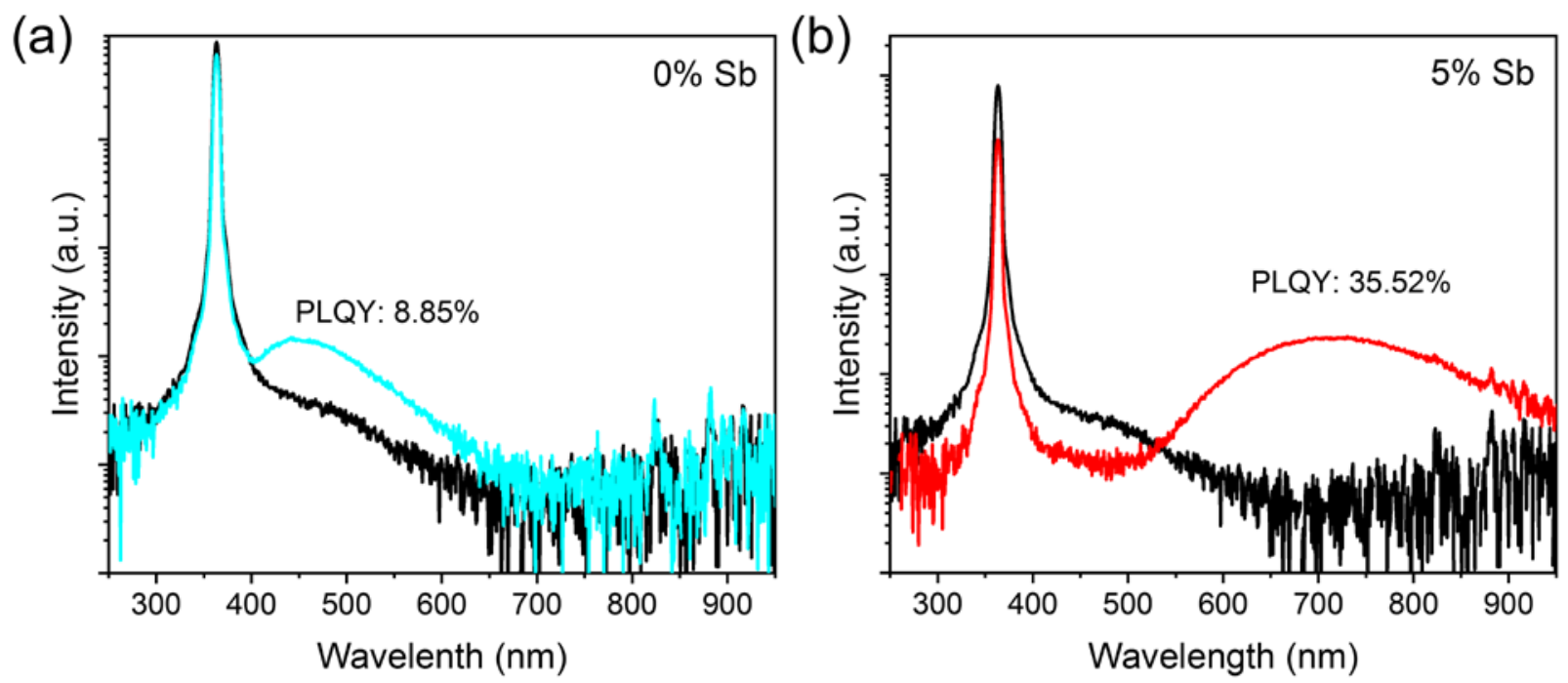

Figure S7. PLQE curves of $\left(\mathrm{C}_{8} \mathrm{NH}_{12}\right)_{6} \mathrm{InBr}_{9} \cdot \mathrm{H}_{2} \mathrm{O}(\mathrm{a})$ and $\left(\mathrm{C}_{8} \mathrm{NH}_{12}\right)_{6} \operatorname{InBr}_{9} \cdot \mathrm{H}_{2} \mathrm{O}: 5 \% \mathrm{Sb}$ (b) upon $365 \mathrm{~nm}$ excitation. 


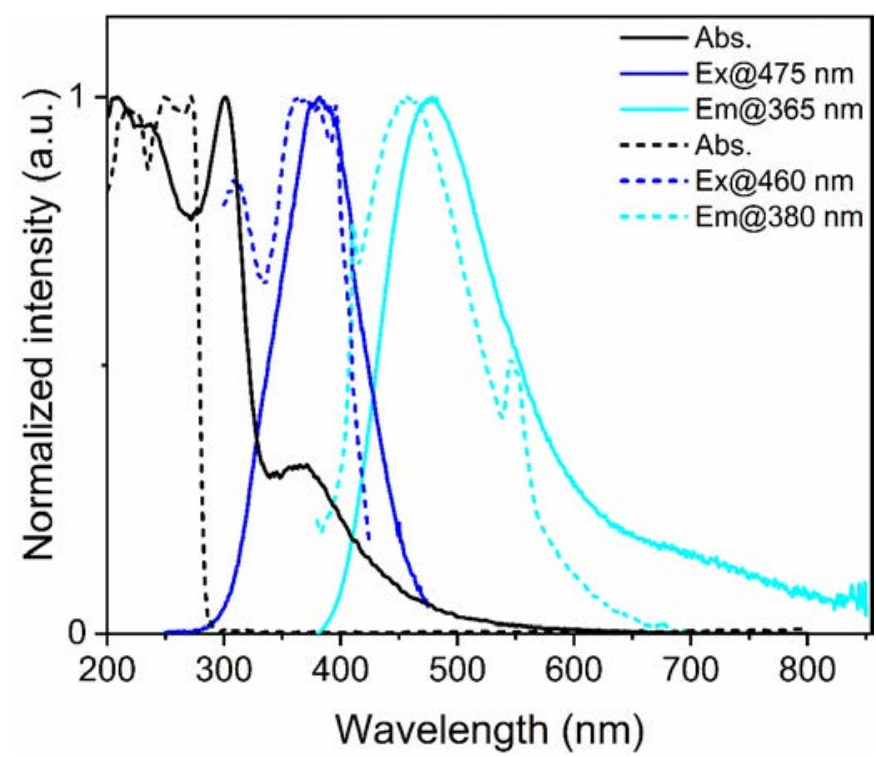

Figure S8. Comparison of absorption, excitation and emission spectra between $\left(\mathrm{C}_{8} \mathrm{NH}_{12}\right)_{6} \mathrm{InBr}_{9} \cdot \mathrm{H}_{2} \mathrm{O}($ solid line) and $\mathrm{C}_{8} \mathrm{NH}_{12} \mathrm{Br}$ salt (dashed line).

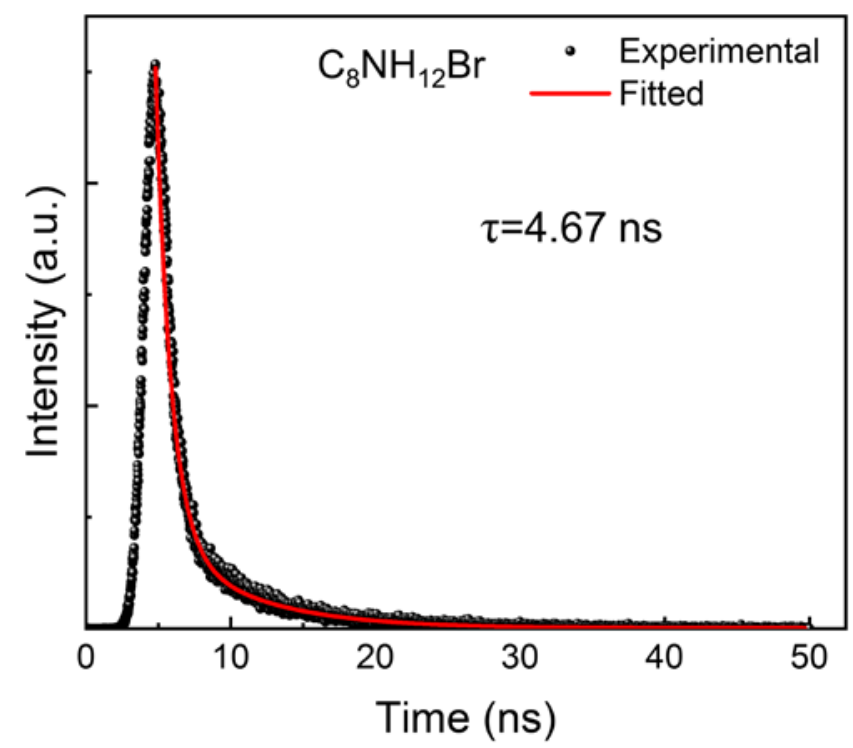

Figure S9. Time-resolved decay curves monitored at $475 \mathrm{~nm}$ of the corresponding organic salt $\mathrm{C}_{8} \mathrm{NH}_{12} \mathrm{Br}$. 
(a)

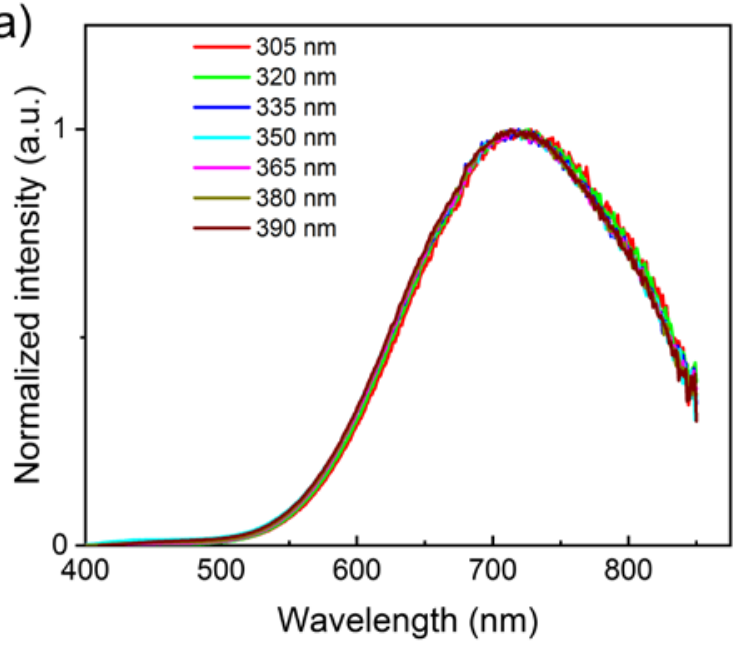

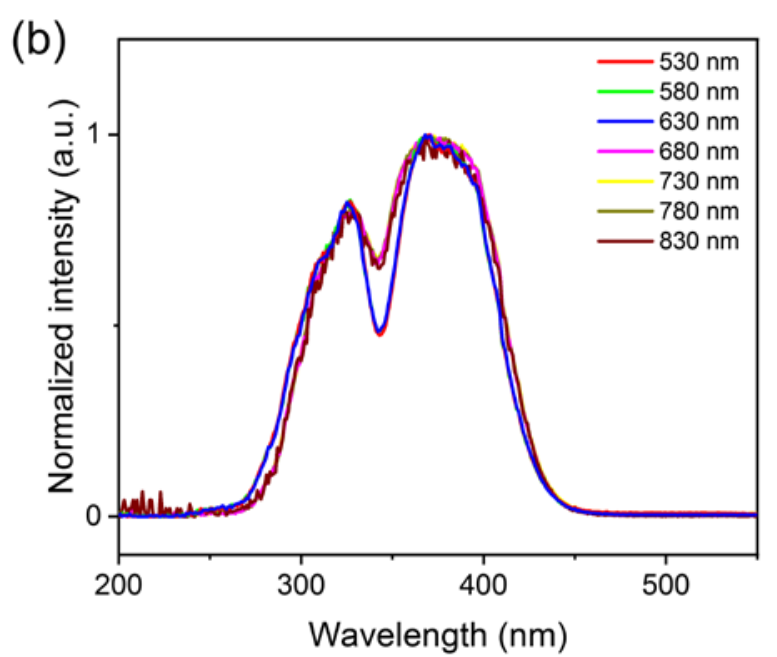

Figure S10. Normalized emission spectra upon different excitation wavelength $(305-390 \mathrm{~nm})$ (a) and Normalized excitation spectra monitored at different emission wavelength $(530-830 \mathrm{~nm})$ (b) of $\left(\mathrm{C}_{8} \mathrm{NH}_{12}\right)_{6} \mathrm{InBr}_{9} \cdot \mathrm{H}_{2} \mathrm{O}: 5 \% \mathrm{Sb}$.

(a)

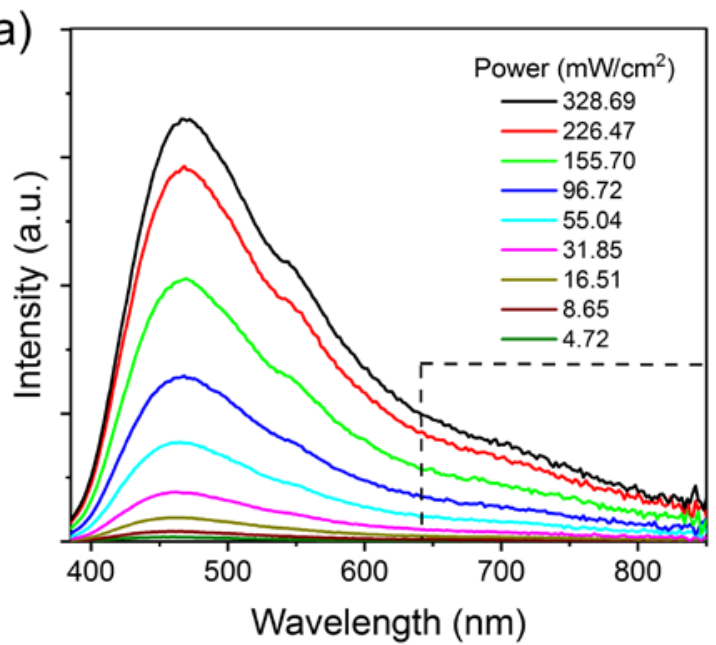

(c)

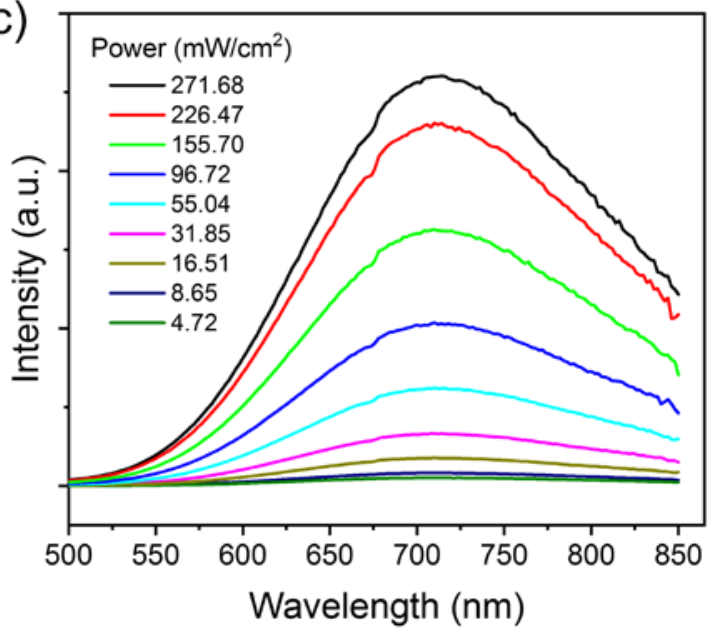

(b)

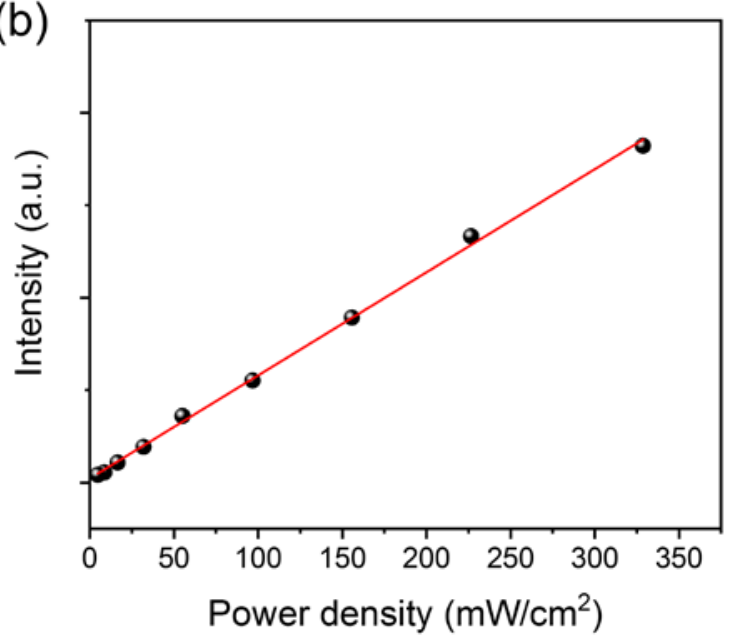

(d)

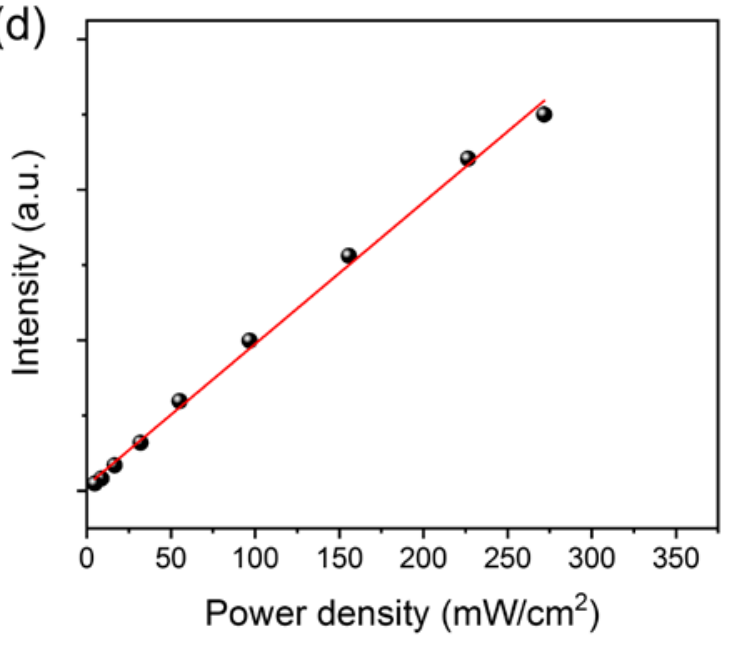

Figure S11. (a, c) Emission spectra versus excitation power density for $\left(\mathrm{C}_{8} \mathrm{NH}_{12}\right)_{6} \mathrm{InBr}_{9} \cdot \mathrm{H}_{2} \mathrm{O}$ and $\left(\mathrm{C}_{8} \mathrm{NH}_{12}\right)_{6} \mathrm{InBr}_{9} \cdot \mathrm{H}_{2} \mathrm{O}: 5 \% \mathrm{Sb}$ at room temperature. (b, d) Emission intensity versus excitation power of red 
emission tail for $\left(\mathrm{C}_{8} \mathrm{NH}_{12}\right)_{6} \mathrm{InBr}_{9} \cdot \mathrm{H}_{2} \mathrm{O}$ and broad emission band for $\left(\mathrm{C}_{8} \mathrm{NH}_{12}\right)_{6} \mathrm{InBr}_{9} \cdot \mathrm{H}_{2} \mathrm{O}: 5 \% \mathrm{Sb}$ at room temperature.

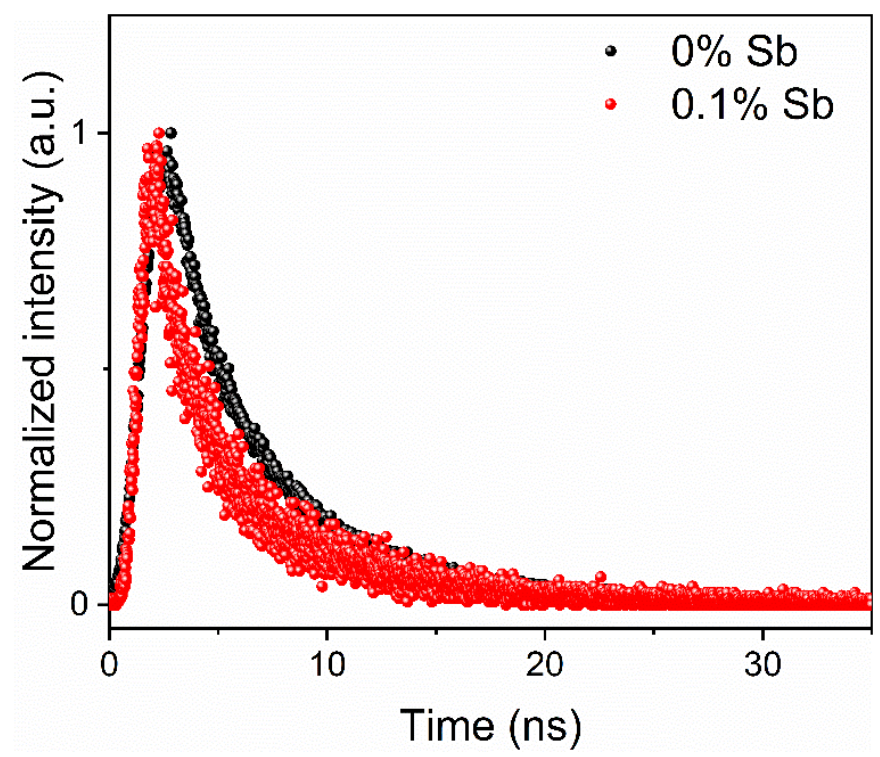

Figure S12. Photoluminescence decay curves of pure $\left(\mathrm{C}_{8} \mathrm{NH}_{12}\right)_{6} \mathrm{InBr}_{9} \cdot \mathrm{H}_{2} \mathrm{O}$ and $\left(\mathrm{C}_{8} \mathrm{NH}_{12}\right)_{6} \mathrm{InBr}_{9} \cdot \mathrm{H}_{2} \mathrm{O}: 0.1 \% \mathrm{Sb}$ monitored at $475 \mathrm{~nm}$.

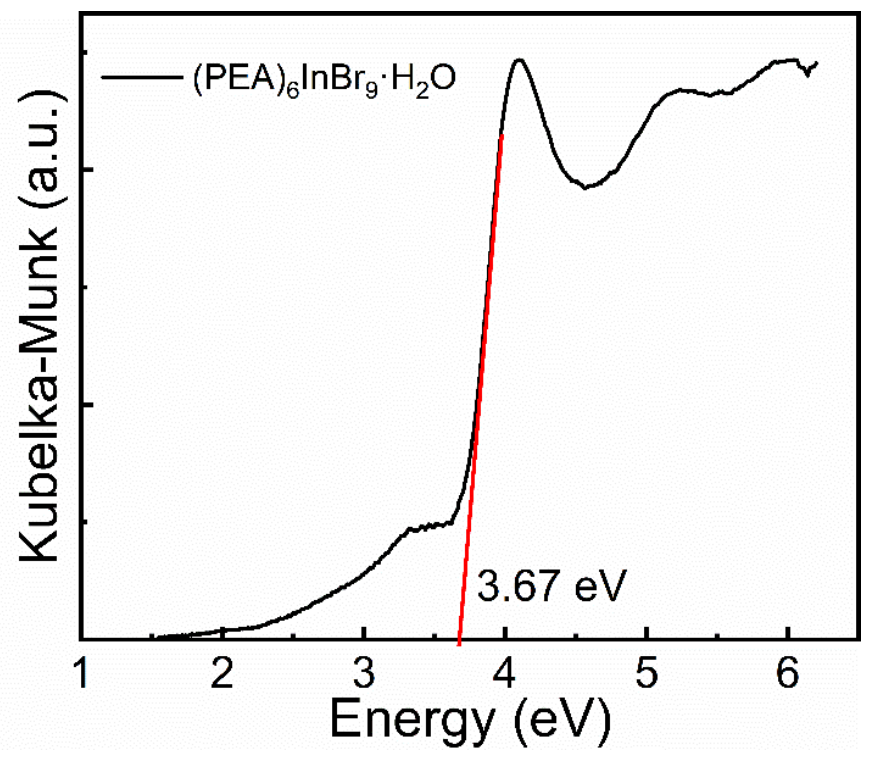

Figure S13. Optical absorption spectra showing bandgap of $\left(\mathrm{C}_{8} \mathrm{NH}_{12}\right)_{6} \mathrm{InBr}_{9} \cdot \mathrm{H}_{2} \mathrm{O}$. 


\section{REFERENCES}

(1) Payne, M. C.; Teter, M. P.; Allan, D. C.; Arias, T. A.; Joannopoulos, J. D., Iterative Minimization Techniques for Ab Initio Total-Energy Calculations: Molecular Dynamics and Conjugate Gradients. Rev. Mod. Phys. 1992, 64, (4), 1045-1097.

(2) Perdew, J. P.; Zunger, A., Self-Interaction Correction to Density-Functional Approximations for Many-Electron Systems. Phys. Rev. B 1981, 23, (10), 5048-5079.

(3) Chadi, D. J., Special Points for Brillouin-Zone Integrations. Phys. Rev. B 1977, 16, (4), 1746-1747. 